\title{
Formation of a Global Contract Research Organization Council for Bioanalysis
}

\author{
"The Global Contract Research Organizations Council (GCC) is proposed to be a global independent group \\ specifically focused on harmonization of bioanalytical guidelines that have particular relevance to scientific bioanalytical \\ issues impacting contract research organizations."
}

Noel Premkumar', Stephen Lowes ${ }^{2}$, James Jersey ${ }^{3}$, Fabio Garofolo ${ }^{\dagger 4}$, Isabelle Dumont ${ }^{4}$ Robert Masse $^{5}$, Betty Stamatiou ${ }^{5}$, Maria C Caturla ${ }^{6}$, Ray Steffen ${ }^{7}$, Michele Malone ${ }^{8}$, Elliot Offman ${ }^{9}$, Timothy Samuels ${ }^{10}$, Phillip Oldfield ${ }^{10}$, Lorella Di Donato", Douglas Fast ${ }^{12}$, Daniel Tang ${ }^{13}$, Marc Moussallie ${ }^{14}$, John Doughty ${ }^{14}$, Mario Rocci ${ }^{15}$, Mike Buonarati ${ }^{16}$, Dominique Gouty ${ }^{17}$, Darioush Dadgar ${ }^{18}$, John Stamatopoulos ${ }^{19}$, Alan Breau ${ }^{20}$, Bernard Ntsikoussalabongui ${ }^{20}$, Mohammed Bouhajib ${ }^{21}$, Bob Nicholson ${ }^{22}$, Richard Tacey ${ }^{22}$, Peter Ketelaar ${ }^{23}$, Chad Briscoe ${ }^{23}$, Shane Karnik ${ }^{24}$, Jaap Wieling ${ }^{25}$, Jon Kirk Smith ${ }^{26}$, Michael J Reid ${ }^{27}$, Richard LeLacheur ${ }^{28}$, John Chapdelaine ${ }^{29}$, Saadya Fatmi ${ }^{29}$, Farhad Sayyarpour ${ }^{30}$, Xinping Fang ${ }^{31}$, Jeremy Cook ${ }^{32}$ \& David Browne ${ }^{32}$

${ }^{\dagger}$ Author for correspondence: Algorithme Pharma Inc., 575 Armand-Frappier Blvd, Laval (Montreal) Quebec, H7V 4B3, Canada Tel.: +I 450973 6077; Fax: +I 450973 2446; E-mail: fgarofolo@algopharm.com

Author affiliations can be found on page 1800

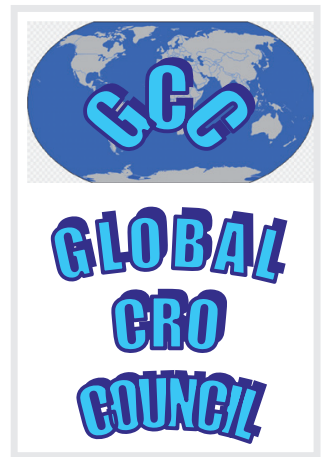

Keywords: bioanalysis $\approx$ bioanalytical CRO = bioanalytical guidelines $₫$ bioanalytical harmonization $\approx$ bioanalytical issues $\approx$ contract research organization $\approx \mathrm{GCC} \approx$ Global CRO Council

\section{Background}

Over the last year, the bioanalytical community strongly expressed their need for international harmonization of bioanalytical guidances through numerous international meetings and publications, and this need was acknowledged by several regulatory agencies [1-5]. Following the 4th Calibration and Validation Group (CVG) Workshop on Regulated Bioanalysis hosted in Montreal (April 2010), a unanimous consensus was reached for the global bioanalytical community to identify non-prescriptive, sciencebased language that could form the basis of a proposed guidance document on bioanalysis [6]. Ideally, such guidance language would describe the rationale behind each bioanalytical requirement and would be presented for consideration by agencies and industry worldwide.

A recent concrete action taken towards such global harmonization of bioanalytical guidances was the creation of the Global Bioanalysis Consortium (GBC). Drawing from representatives of scientific associations with involvement in regulated bioanalysis across the globe, the objective of the GBC is to merge existing or emerging bioanalytical guidances and create a unified document that can be presented to the regulatory authorities in various countries and regions. The development of the GBC is currently in process, with the intention to present plans and updates at upcoming bioanalytical meetings and to seek input from the scientists conducting bioanalysis [7].

At the 4th CVG Bioanalysis Workshop in Montreal, Canada, several Contract Research Organizations (CROs) highlighted the importance of having a strong and cohesive CRO contribution to the global harmonization process. Having CROs, academic laboratories and pharmaceutical industries appropriately represented was viewed as critical to identifying optimum language. Consequently, the proposal to build a Global CRO Council (GCC) arose, designed to be a distinct group consisting exclusively of bioanalytical CRO members conducting regulated bioanalysis business.

To initiate creation of the GCC, a special closed forum was held in Montreal on 14 September 2010, where 41 executive representatives from 32 bioanalytical CROs were present to discuss scientific harmonization issues and the institution of the GCC. This first CRO

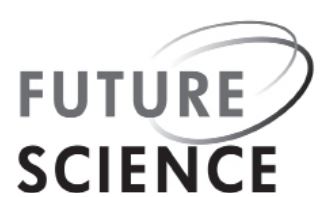


closed forum was a unique occasion to discuss, review and share perspectives upon a unified CRO approach on how to support the global harmonization of bioanalytical guidances.

\section{Harmonization issues}

Bioanalytical CROs work under strict regulatory standards, and since they operate globally in various countries, similar to their pharmaceutical and biotechnology clients, they have to deal with different regulations and guidances. The differences in the guidance documents (or lack of) from major regulatory agencies (e.g., the FDA for USA, EMA for Europe, ANVISA for Brazil, TGA for Australia, SFDA for China and MHLW for Japan) are becoming a serious hurdle for CROs that conduct studies destined for submission to different health agencies. Many CROs performing bioequivalence studies are struggling to comply with both national and international guidelines, especially in emerging economies such as Asia-Pacific, Latin America and Eastern Europe. Therefore, global harmonization of bioanalytical guidances would have a strong, profound and positive impact on how CROs function in such a global and regulated environment, and would help to achieve a consistent bioanalytical quality standard.

\section{“...global harmonization of bioanalytical guidances would have a strong, profound and positive impact on how contract research organizations function \\ in such a global and regulated environment, and would help to achieve $a$ consistent bioanalytical quality standard."}

Several specific challenges that CROs are facing were raised and discussed during the first CRO closed forum. For instance, multiple questions regarding cross-validation of transferred methods from sponsor laboratories and associated stability assessments still need consensus. Adequate guidance and quality standards are lacking for some matrix-related issues, including the assessment of hemolysis and the use of matrices from different ethnic groups. This is also the case for many other general validation requirements concerning sample re-injection versus re-assay, investigations on incurred sample reproducibility (ISR) failures; re-assay for pharmacokinetic reasons; assays using dried blood spot analysis; and method parameters for biomarkers/ligand-binding assays. In addition, suitable standards outlining an appropriate Quality Management System for bioanalytical laboratories have not yet been issued by most of the major regulatory agencies. This is a small sampling of topics and not considered to be an exhaustive list.

\section{Formation of the Global CRO Council}

The GCC is proposed to be a global independent group specifically focused on harmonization of bioanalytical guidelines that have particular relevance to scientific bioanalytical issues impacting CROs. The extended vision of the GCC is to present a formal and cohesive CRO body to global health authorities and regulatory agencies around the world to help address the specific scientific challenges $\mathrm{CROs}$ encounter in conducting regulated bioanalysis. The goals and objectives of the GCC include the following:

- Offer a forum for the CRO community to gather input and reach a consensus on concerns about regulatory developments in bioanalysis and CRO-specific challenges;

- Communicate with other bioanalytical associations (e.g., the American Association of Pharmaceutical Scientists [AAPS], the CVG and the European Bioanalysis Forum [EBF]) and with regulatory agencies via official publications and presentations at bioanalytical meetings;

- Generate a scientific document to be presented to the GBC that will provide input from a $\mathrm{CRO}$ perspective in an effort to achieve global harmonization of bioanalytical guidances;

- Support the interests of CRO sponsors through the identification of specific solutions to bioanalytical regulatory body concerns.

When building the GCC, the CROs will be considered as the members, in other words, the CRO itself will be a member, not the individual person representing the CRO. One or two representatives will represent each CRO, and these representatives must be part of the CRO's senior management. The structure of the GCC will be established in a way that will promote collaboration, facilitate effective communication and maintain efficiency in reaching science-driven consensus.

Since the GCC members are competitors often sharing the same clients, all members will have equal representation. Hence, a flat 
organizational structure that preserves equality and minimizes hierarchy is advocated. The GCC must not interfere with the competitive environment CROs operate in. Therefore, the operation of the GCC must officially discourage any potential anti-competitive behavior and totally avoid any operational, strategic, pricing or confidential issues. A formal articulation of the GCC's mission in by-laws is being considered, as well as the creation of admonition statements and meeting minutes for each GCC meeting. In addition, specific GCC members will work together on the incorporation of the GCC as a nonprofit independent international organization.

\section{"Since the Global CRO Council members are competitors often sharing the same clients, all members will have equal representation."}

As previously outlined, effective communication will be crucial to the success of the GCC. This will be accomplished internally using face-to-face dialog, electronic conversations and surveys. External visibility of the GCC will be supported by official publications, presentations and involvement in cross-organization discussion groups. A GCC closed forum will be held each year, in parallel with a major scientific conference that CROs will usually plan to attend (e.g., the AAPS, CVG and EBF conferences). Other special GCC closed forums may also be organized as needed; for example, following particular events or emerging issues needing appropriate response from the CRO perspective. To ensure efficiency, the GCC will need to focus on clear goals, outcomes and action plans during GCC meetings. Figure I summarizes some of the dynamics surrounding the GCC.

Only GCC members (i.e., CRO representatives) who are present at a particular GCC closed forum will take part in the decision-making process, utilizing the following guidelines:

- Inclusive: involving as many GCC member companies as possible, from various countries and regions;

- Participatory: actively soliciting the input and the participation of all GCC members;

- Cooperative: working to reach the most appropriate decision for the group, rather than pursuing a majority opinion potentially to the detriment of a minority;

- Egalitarian: all GCC members having equal input;

- Solution-oriented: GCC members aiming for common agreement over differences and reaching effective decisions using mutually beneficial solutions;

- Dissent early: maximizing chances of accommodating views.

The fact that the GCC is global and not local is most certainly one of its most essential features, and this will need to be kept in mind throughout the building and growing of the GCC.

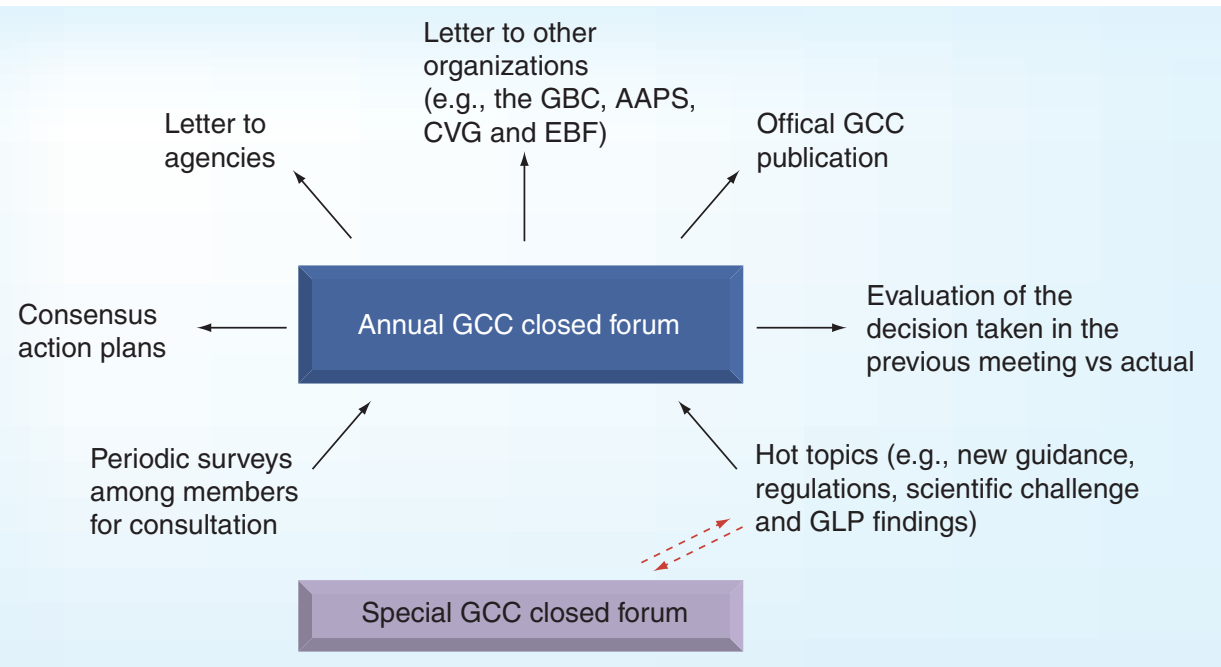

Figure 1. Global contract research organizations council dynamics. AAPS: American Association of Pharmaceutical Scientists; CRO: Contract research organization; CVG: Calibration and Validation Group; EBF: European Bioanalysis Forum; GBC: Global Bioanalysis Consortium; GCC: Global CRO Council; GLP: Good Laboratory Practices. 


\section{Future considerations}

In the upcoming months, the GCC members will continue working together on the institution of the GCC and on the approach to adopt in communicating with regulatory agencies. The next annual GCC closed forum is planned to take place in Montreal on 15 April 2011, immediately following the 5th CVG Workshop on Bioanalysis in Montreal (11-14 April 2011). The subsequent GCC closed forum is tentatively scheduled to occur concurrently with the October 2011 AAPS conference (Washington, USA) or the December 2011 EBF conference (Barcelona, Spain). Anyone who can provide relevant insight, wisdom or knowledge in relation to the building of a group such as the GCC is invited to provide input and feedback.

Financial \& competing interests disclosure

The authors have no relevant affiliations or financial involvement with any organization or entity with a financial interest in or financial conflict with the subject matter or materials discussed in the manuscript. This includes employment, consultancies, honoraria, stock ownership or options, expert testimony, grants or patents received or pending, or royalties. No writing assistance was utilized in the production of this manuscript.

\section{Author affiliations}

\author{
'ABC Laboratories \\ ${ }^{2}$ Advion BioServices \\ ${ }^{3}$ Agilux Laboratories \\ ${ }^{4}$ Algorithme Pharma Inc. \\ ${ }^{5}$ Anapharm \\ ${ }^{6}$ Anapharm Europe \\ ${ }^{7} \mathrm{BASi}$ \\ ${ }^{8}$ Worldwide Clinical Trials Drug Development Solutions \\ ${ }^{9}$ Celerion \\ ${ }^{10} \mathrm{Charles}$ River \\ "CIRION \\ ${ }^{12}$ Covance Laboratories \\ ${ }^{13}$ Frontage Laboratories \\ ${ }^{14}$ Huntingdon Life Sciences \\ ${ }^{15}$ CON Development Solutions \\ ${ }^{16}$ Intertek Alta LCMS \\ ${ }^{17}$ Intertek Alta Immunochemistry \\ ${ }^{18} \mathrm{KCAS}$ \\ ${ }^{19} \mathrm{LAB}$ Research \\ ${ }^{20} \mathrm{MPI}$ Research \\ ${ }^{2}$ Pharma Medica Research \\ ${ }^{22} \mathrm{PPD}$ \\ ${ }^{23}$ PRA International \\ ${ }^{24}$ Pyxant Labs \\ ${ }^{25}$ QPS, LLC \\ ${ }^{26}$ Smithers Pharma Services \\ ${ }^{27}$ SNBL USA \\ ${ }^{28}$ Taylor Technology \\ ${ }^{29}$ Warnex Bioanalytical Services \\ ${ }^{30}$ WIL Research Laboratories \\ ${ }^{31}$ XenoBiotic Laboratories (XBL) \\ ${ }^{32}$ York Bioanalytical Solutions
}

\section{Bibliography}

1 Lausecker B, van Amsterdam P, Brudny-Kloeppel M, Luedtke S, Timmerman P. European Bioanalysis Forum and the way forward towards harmonized regulations. Bioanalysis 1(5), 873-875 (2009).

2 Abbott R. European Bioanalysis Forum, 2nd Open Symposium: The broadening scope of validation: towards best practices in the world of bioanalysis. Bioanalysis 2(4), 703-708 (2010).
3 Timmerman P, Lowes S, Fast DM, Garofolo F. Request for global harmonization of the Guidance for Bioanalytical Method Validation and Sample Analysis. Bioanalysis 2(4), 683 (2010).

4 Bansal S, Arnold M, Garofolo F. International harmonization of bioanalytical guidance. Bioanalysis 2(4), 685-687 (2010).

5 van Amsterdam P, Lausecker B, Luedtke S, Timmerman P, Brudny-Kloeppel M. Towards harmonized regulations for bioanalysis: moving forward! Bioanalysis 2(4), 689-691 (2010)
6 Savoie N, Garofolo F, van Amsterdam P et al. 2010 White paper on recent issues in regulated bioanalysis and global harmonization of bioanalytical regulations - from The 4th Calibration and Validation Group Workshop. Bioanalysis DOI:10.4155/BIO.10.164 , (2010) (In Press).

7 van Amsterdam P, Arnold M, Bansal S et al. Building the Global Bioanalysis Consortium: working towards a functional globally acceptable and harmonized guideline on bioanalytical method validation. Bioanalysis 2(11), 1801-1803 (2010). 